\title{
Lithium-Salt Mediated Synthesis of a Covalent Triazine Framework for Highly Stable Lithium Metal Batteries
}

\author{
Tianhong Zhou , Yan Zhao ${ }^{+}$, Jang Wook Choi, ${ }^{*}$ and Ali Coskun*
}

\begin{abstract}
A new strategy for the synthesis of a covalent triazine framework (CTF-1) was introduced based on the cyclotrimerization reaction of 1,4-dicyanobenzene using lithium bis(trifluoromethanesulfonyl)imide (LiTFSI) under ionothermal conditions. LiTFSI not only served as a catalyst, but also facilitated the in situ generation and homogeneous distribution of LiF particles across the framework. The hierarchical structure resulting upon integration of CTF-LiF onto an airlaid-paper $(A P)$ offered unique features for lithium metal anodes, such as lithiophilicity from $C T F$, interface stabilization from LiF, and sufficient lithium storage space from AP. Based on this synergistic effect, the AP-CTF-LiF anode exhibited stable cycling performance even at a current density of $10 \mathrm{~mA} \mathrm{~cm}^{-2}$.
\end{abstract}

W th growing demand of rechargeable high energy density batteries, lithium $(\mathrm{Li})$ metal anodes have aroused discernable attention due to their unparalleled electrochemical properties: the lowest electrochemical potential $(-3.04 \mathrm{~V}$ vs. standard hydrogen electrode) and the highest theoretical specific capacity $\left(3860 \mathrm{mAhg}^{-1}\right) \cdot{ }^{[1]}$ However, uncontrolled dendrite growth at the surface has impeded the advancement of $\mathrm{Li}$ metal anodes for their integration into practical cell settings. Continuous dendrite growth gives rise to an unstable solidelectrolyte interphase (SEI) layer, leading to a poor Coulombic efficiency (CE) in each cycle and an excessive consumption of the electrolyte. The uncontrolled dendrite growth also causes internal short-circuits, thus boosting the possibility of fire hazard. ${ }^{[2]}$

Numerous approaches have been proposed to address issues associated with $\mathrm{Li}$-dendrite growth. A variety of electrolyte additives were introduced, including $\mathrm{Cs}^{+}$and $\mathrm{Rb}^{+},{ }^{[3]}$ ionic liquids, ${ }^{[4]}$ co-solvents, ${ }^{[5]}$ vinylene carbonate,${ }^{[6]}$ $\mathrm{LiNO}_{3}$ and polysulfides. ${ }^{[7]}$ These additives were used to promote stable SEI layers by enhancing the mechanical properties and the Li-ion diffusivity or manipulating electrostatic fields near the $\mathrm{Li}$ electrode surface. The addition of

[*] T. Zhou, ${ }^{[+]}$Y. Zhao, ${ }^{[+]}$Prof. A. Coskun

Department of Chemistry, University of Fribourg

Chemin de Musee 9, Fribourg 1700 (Switzerland)

E-mail: ali.coskun@unifr.ch

Prof. J. W. Choi

School of Chemical and Biological Engineering and Institute of

Chemical Processes, Seoul National University

1 Gwanak-ro, Gwanak-gu, Seoul 08826 (Republic of Korea)

E-mail: jangwookchoi@snu.ac.kr

$\left.{ }^{+}\right]$These authors contributed equally to this work. lithium fluoride $(\mathrm{LiF})$ was found to be particularly benefi$\mathrm{cia}^{[8]}$ from the perspective of both, mechanical stability and ionic conductivity. In an attempt to enhance the mechanical stability of SEI layers and Li-host structures, more recently also elastic polymers were incorporated. ${ }^{[9]}$ Besides Li foil, a variety of host structures were developed, such as functionalized nanocarbons ${ }^{[10]}$ and metallic nanomaterials. ${ }^{[11]}$ Furthermore, seed materials were placed inside the host frameworks to drive Li plating in confined volumes by engaging an Lialloying reaction mechanism. ${ }^{[12]}$ The series of remarkable achievements conveys a useful lesson that it would be desirable to develop Li-host structures in a hierarchical platform while utilizing the beneficial effect of $\mathrm{LiF}$.

Covalent triazine frameworks (CTFs), a subclass of porous organic polymers, were firstly synthesized by Thomas and co-workers by trimerization of aromatic nitriles using $\mathrm{ZnCl}_{2}$ under ionothermal conditions. ${ }^{[13]}$ Considering their modularity, relatively high nitrogen contents and promising electrical properties, CTFs were widely applied in gas storage, catalysis, $\mathrm{CO}_{2}$ separation and energy storage. ${ }^{[14]}$ Simultaneously, alternative synthetic strategies have also been developed to obtain CTFs under mild reaction conditions. These strategies include acid-catalyzed approaches, ${ }^{[14 a, 15]}$ polycondensation of aldehydes and amidines under basic conditions ${ }^{[16]}$ and $\mathrm{P}_{2} \mathrm{O}_{5}$-catalyzed conversion of aromatic amides. ${ }^{[17]}$ In particular, the utilization of elemental sulfur and aromatic nitriles to form CTFs points to the versatility of the synthetic strategies. ${ }^{[14 b]}$ LiTFSI has been lately recognized as a useful salt in Li-ion battery electrolytes, as it gives rise to a relatively stable interface with Li metal electrodes. However, its potential to mediate the synthesis of CTFs has never been explored. Herein, we develop a new strategy for the synthesis of CTF-1, that is based on the trimerization of 1,4dicyanobenzene (DCB) using LiTFSI under ionothermal conditions (Figure 1a). Notably, this reaction also leads to the in situ formation of uniformly distributed LiF particles within the framework. Considering its high free volume and lowcost, airlaid-paper (AP) was used as a Li host and further coated with CTF-LiF for interface stabilization of the $\mathrm{Li}$ metal anode. Owing to the synergistic effect of the lithiophilicity of CTF, the interface stabilization by $\mathrm{LiF}$, and the large Li-storage volume of AP, spatially uniform Li plating over the entire electrode was achieved. The Li metal anode based on AP-CTF-LiF showed an excellent cycling performance, such as stable $\mathrm{CE}$ for 220 cycles at $10 \mathrm{~mA} \mathrm{~cm}^{-2}$ and small polarization for 700 cycles at $5 \mathrm{~mA} \mathrm{~cm}^{-2}$.

The synthesis of CTF-LiF was achieved by trimerization of 1,4-dicyanobenzene at $300^{\circ} \mathrm{C}$ using LiTFSI under ionothermal conditions. The lower melting point of LiTFSI $\left(238^{\circ} \mathrm{C}\right)$ compared to that of $\mathrm{ZnCl}_{2}$ enabled the formation 
(a) (b)
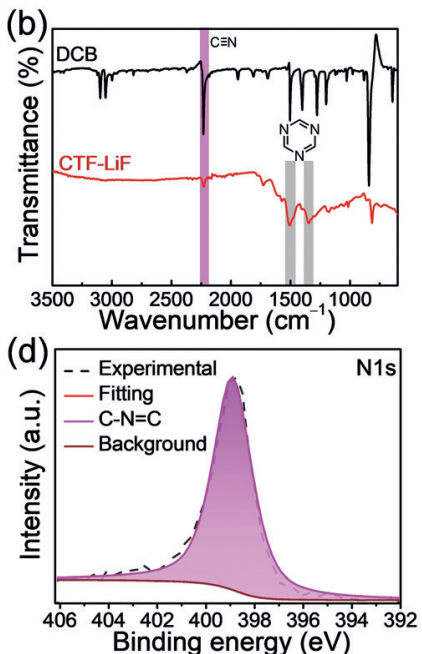

Binding energy $(\mathrm{eV})$
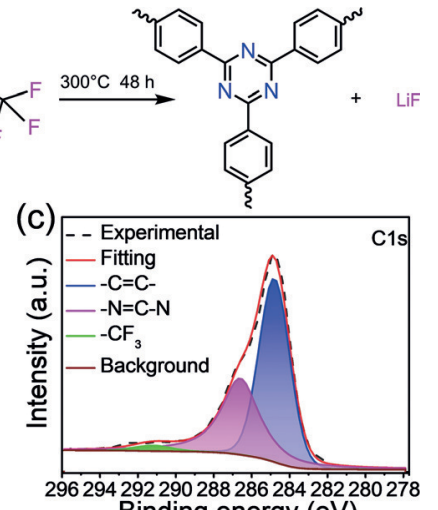

(e)

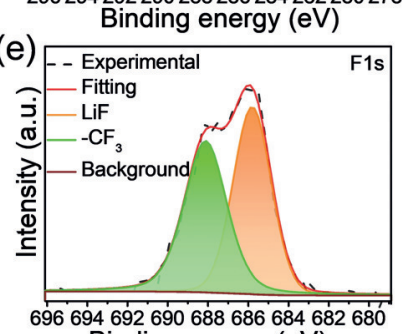

Binding energy $(\mathrm{eV})$
Figure 1. a) Synthesis of CTF-LiF from Li salt and DCB. b) FT-IR spectra of DCB and CTF-LiF. C-e) XPS spectra of CTF-LiF in C 1s, N 1s and $\mathrm{F} 1 \mathrm{~s}$ branches, respectively.

of CTF at a lower temperature through strong Lewis-acid base interactions in the melt-state between LiTFSI and 1,4dicyanobenzene. To our pleasant surprise, the partial degradation of LiTFSI during the reaction led to the in situ formation of $\mathrm{LiF}$ particles, which are uniformly distributed within the CTF. To prove the formation of CTF-LiF, Fourier transform infrared (FT-IR) analysis was performed (Figure $1 \mathrm{~b})$. Notably, the attenuation of a nitrile stretching band at $2228 \mathrm{~cm}^{-1}$ was accompanied by the emergence of triazine peaks at 1506 and $1348 \mathrm{~cm}^{-1}$, thus pointing to the formation of a CTF backbone. ${ }^{[13-14]}$ The structure of CTF-LiF was further elucidated by powder X-ray diffraction (PXRD) analysis, as shown in Figure S1 (see Supporting Information). The PXRD spectrum indicated the presence of a pure LiF phase located within the amorphous CTF. The crystallite size of LiF was calculated by the Scherrer equation to be $36.5 \mathrm{~nm}$. In order to further investigate the chemical state of elements in CTF-LiF, $\mathrm{X}$-ray photoelectron spectroscopy (XPS) was performed. The XPS survey spectrum (Figure S2) of CTF-LiF revealed the presence of carbon, nitrogen, fluorine and lithium. The deconvoluted $\mathrm{C}$ 1s spectrum (Figure 1c) showed three peaks at 284.8, 286.6 and $291.2 \mathrm{eV}$, which can be assigned to aryl carbons $(-\mathrm{C}=\mathrm{C}-),{ }^{[14 \mathrm{~b}]}$ carbon atoms in the triazine ring $(\mathrm{N}=\mathrm{C}-\mathrm{N}),{ }^{[14 \mathrm{~b}]}$ and lastly carbon atoms of the remaining Li salt $\left(-\mathrm{CF}_{3}\right){ }^{[18]}$ respectively. As for the XPS N 1s spectrum (Figure $1 \mathrm{~d}$ ), it showed a single peak at $398.9 \mathrm{eV}$, which is in accordance to previous reports on $\mathrm{CTFs}^{[14 \mathrm{a}]}$ and was attributed to nitrogen atoms in the triazine ring. The XPS F 1s spectrum (Figure 1e) revealed two peaks at 685.8 and $688.1 \mathrm{eV}$, which corresponds to $\mathrm{LiF}^{[19]}$ and LiTFSI, ${ }^{[18]}$ respectively. ${ }^{13} \mathrm{C}$ magic angle spinning (MAS) solid-state nuclear magnetic resonance (ssNMR) analysis was conducted to prove the molecular connectivity of CTF-LiF (Figure S3).
The signal at $170 \mathrm{ppm}$ is assigned to carbon atoms of the triazine ring, while the other strong signals at 138 and 130 ppm correspond to the phenyl ring. ${ }^{[20]}$ Brunauer-EmmettTeller (BET) surface area analysis was carried out to analyze the porosity of CTF-LiF (Figure S4), which is found to be non-porous due to the blockage of pores by in situ grown LiF particles as well as the residual LiTFSI. Thermogravimetric analysis (TGA) of CTF-LiF showed good thermo-oxidative stability under air atmosphere (Figure S5). In order to identify the CTF:LiF ratio, we performed a control experiment by heating the same amount of LiTFSI under the same experimental conditions. Upon the completion of this control experiment, the pure $\mathrm{LiF}$ phase was identified by PXRD (Figure S6 and S7) and its corresponding weight was found to be $15 \mathrm{mg}$. The larger crystallite size ( 1 to $10 \mu \mathrm{m}$ ) in the control LiF sample proved the templating role of the CTF to tune the crystal size. Based on the quantity of CTF-LiF (65 mg), we predicted that the ratio of CTF:LiF is about $3.3: 1$ by weight. In addition, elemental analysis (EA) was conducted to identify the elemental composition of CTF-LiF. CTF-LiF contained $54.57 \mathrm{wt} \%$ carbon, $2.09 \mathrm{wt} \%$ hydrogen and $19.72 \mathrm{wt} \%$ nitrogen (Table S1). These results are in good agreement with the theoretical ratio and also with the CTF:LiF ratio calculated above. Notably, lowering the reaction temperature allowed us to achieve the near ideal nitrogen content (Table S2) in CTF-1, an essential parameter for high lithiophilicity. The morphology and elemental distribution of CTF-LiF were studied by field emission scanning electron microscopy (FE-SEM). CTF-LiF exhibited irregular particle distribution with sizes ranging from 2 to $10 \mu \mathrm{m}$ (Figures S8a and S8d). Elemental mapping of nitrogen and fluorine atoms in CTF-LiF by energy-dispersive X-ray spectroscopy (EDS) analysis verified the uniform distribution of LiF within CTF (Figure S9).

High lithiophilicity of CTF, coupled with the presence of $\mathrm{LiF}$, prompted us to investigate CTF-LiF as an active Lihosting material for Li metal anodes. We identified commercial AP (45 wt \% polyester and $55 \mathrm{wt} \%$ cellulose) as a 3D scaffold platform in order to store a substantial amount of Li. The SEM analysis of AP showed a randomly oriented 3D fiber structure with large internal voids (Figures S8b and S8e). In order to further induce the stable Li nucleation inside the AP, CTF-LiF was adsorbed onto the AP (AP-CTF-LiF) with a loading of $0.2 \mathrm{mg} \mathrm{cm}^{-2}$. As shown in Figures S8c and S8f, a uniform distribution of CTF-LiF particles was observed on the AP fiber surface.

In order to investigate the synergistic effect of AP-CTF$\mathrm{LiF}$ on the suppression of Li-dendrite growth, repeated cycles of Li plating and stripping at $2 \mathrm{mAh} \mathrm{cm}^{-2}$ were imposed for bare $\mathrm{Cu}, \mathrm{AP}$ and AP-CTF-LiF electrodes by using Li metal foil as the counter electrode (Figure 2). Compared to the significant amount of Li-dendrite growth on bare $\mathrm{Cu}$ foil (Figure 2a and Figure S10a), the incorporation of AP could decrease the formation of dendrites to some extent. However, uneven Li plating within the AP-skeleton was still observed (Figure 2b). In contrast, the AP-CTF-LiF layer could guide the $\mathrm{Li}$ ions to form uniform $\mathrm{Li}$ metal deposits in the entire electrode host (Figure 2c). Both AP and AP-CTF-LiF electrodes showed only the $3 \mathrm{D}$ scaffold structures, pointing 

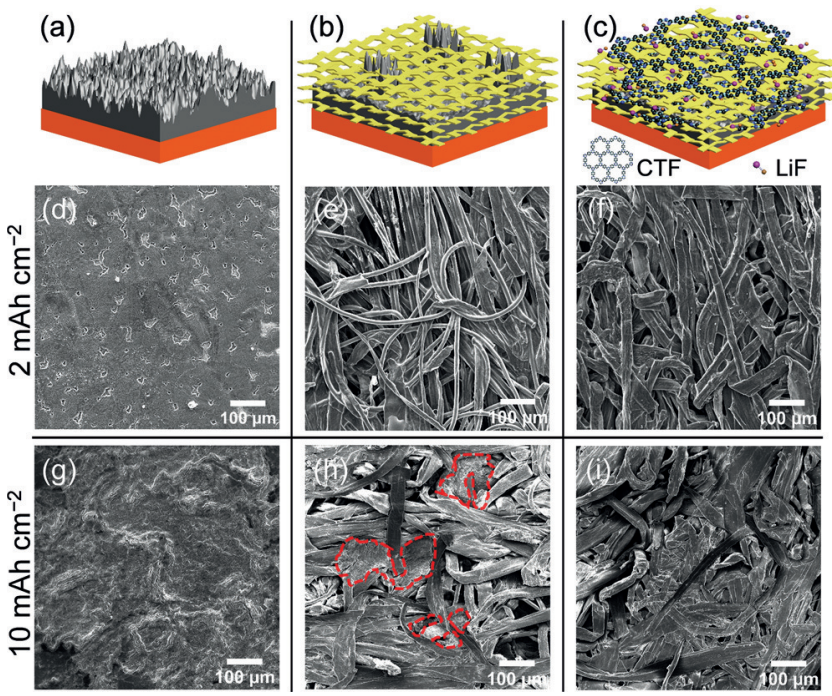

Figure 2. $\mathrm{a}-\mathrm{c}$ ) Bare $\mathrm{Cu}, \mathrm{AP}$ and AP-CTF-LiF electrodes upon Li plating. d-f) SEM images of bare Cu, AP and AP-CTF-LiF electrodes after $2 \mathrm{mAh} \mathrm{cm}^{-2}$ of Li plating. g-i) SEM images of bare $\mathrm{Cu}, \mathrm{AP}$ and AP-CTF-

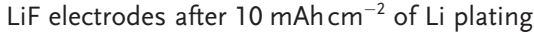

to the fact that $\mathrm{Li}$ metal was well stored within the hierarchical scaffolds (Figure S10b,c). After increasing the $\mathrm{Li}$ plating current rate to $10 \mathrm{mAhcm}^{-2}$ (Figure $2 \mathrm{~g}-\mathrm{i}$ ), $\mathrm{Li}$ deposits on the bare $\mathrm{Cu}$ foil showed many cracks, owing to nonuniform Li plating (Figure S10d). As for the AP, small islands of Li metal were still observed (Figure S10e) on the surface (highlighted in red in Figure $2 \mathrm{~h}$ ), which may ultimately lead to the formation of "dead" Li upon cycling. In contrast, AP-CTF-LiF still maintained the pure 3D framework structure without any obvious Li metal deposits on the surface (Figure S10f), further demonstrating the critical role of CTF-LiF to induce uniform Li nucleation and plating in the scaffold.

In order to further demonstrate the ability of CTF-LiF for stable cycling as Li metal anodes, $\mathrm{CEs}$ of bare $\mathrm{Cu}$, AP and APCTF-LiF were evaluated (Figure $3 \mathrm{a}-\mathrm{c}$ ). For $1 \mathrm{mAh} \mathrm{cm}^{-2}$ of plating/stripping, AP-CTF-LiF showed a very stable CE of $97.3 \%$ for 240 cycles at $3 \mathrm{~mA} \mathrm{~cm}^{-2}$ and $96.2 \%$ for 220 cycles at $10 \mathrm{~mA} \mathrm{~cm}^{-2}$. In contrast, AP exhibited inferior cycling performances with CEs of $96.9 \%$ for 85 cycles (at $3 \mathrm{mAcm}^{-2}$ ) and $92.4 \%$ for 60 cycles (at $10 \mathrm{mAcm}^{-2}$ ). For bare $\mathrm{Cu}$, it showed serious fluctuations of the $\mathrm{CE}$ at both, 3 and $10 \mathrm{~mA} \mathrm{~cm}^{-2}$, owing to the formation of short-circuits from uncontrolled $\mathrm{Li}$-dendrite growth. When increasing the areal capacity to $2 \mathrm{mAh} \mathrm{cm}^{-2}$ at a current rate of $1 \mathrm{mAcm}^{-2}$ (Figure $3 \mathrm{c}$ ), AP-CTF-LiF could also exhibit a superior CE stability of $98.3 \%$ for 160 cycles, while AP showed $98.4 \%$ for 80 cycles and $\mathrm{Cu}$ exhibited $98.4 \%$ only for 40 cycles. Even under relatively harsh conditions, such as a current density of $6 \mathrm{~mA} \mathrm{~cm}^{-2}$ with $3 \mathrm{mAh} \mathrm{cm}^{-2}$, AP-CTF-LiF still showed the best stability of the CE, sustaining over 60 cycles (Figure S11, Table S3).

To clearly demonstrate the influence of the CTF backbone, we designed a control experiment by loading only the same amount of LiF (also synthesized from LiTFSI) on AP. When AP, AP-CTF-LiF and AP-LiF (see Figure S12) were
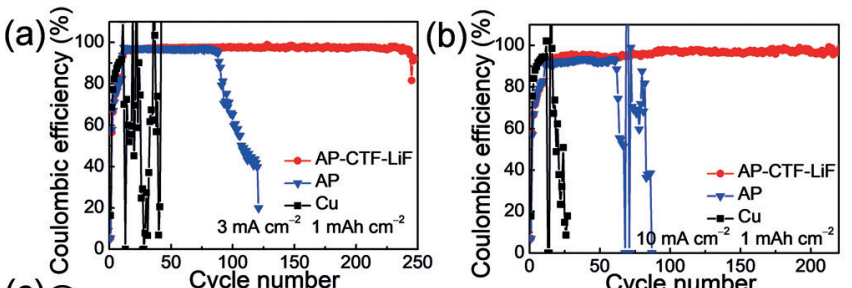

(c)
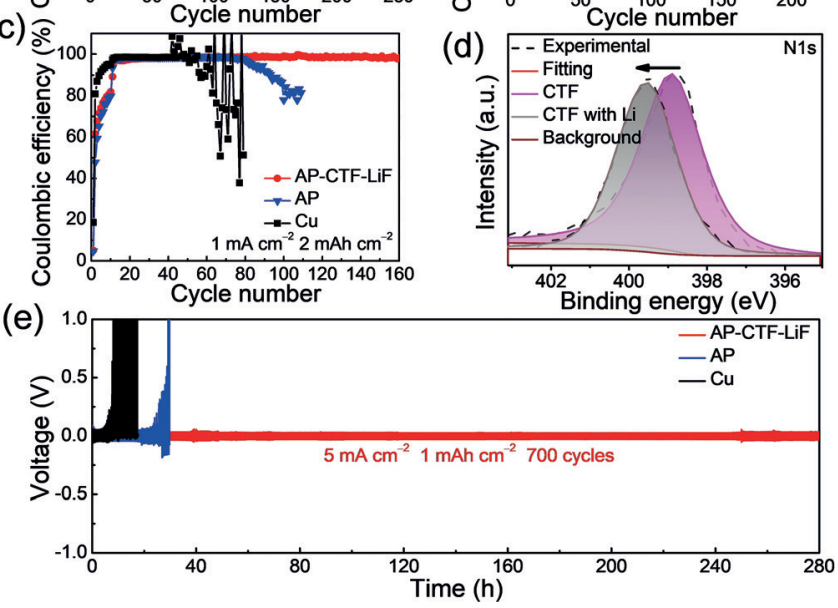

Figure 3. a-c) CE stability over cycling for bare Cu, AP and AP-CTF-LiF electrodes during repeated Li plating/stripping: a) Current density of $3 \mathrm{mAcm}^{-2}$ with areal capacity of $1 \mathrm{mAh} \mathrm{cm}^{-2}$, b) current density of $10 \mathrm{mAcm}^{-2}$ with areal capacity of $1 \mathrm{mAh} \mathrm{cm}^{-2}$, c) current density of $1 \mathrm{mAcm}^{-2}$ with areal capacity of $2 \mathrm{mAhcm}^{-2}$. d) XPS $\mathrm{N} 1 \mathrm{~s}$ spectra of raw CTF and CTF with Li plated inside. e) Voltage-time profiles of symmetric cells with bare Cu, AP and AP-CTF-LiF electrodes when measured at $5 \mathrm{mAcm}^{-2}$ with $1 \mathrm{mAhcm}^{-2}$.

investigated at $1 \mathrm{mAh} \mathrm{cm}^{-2}$ of $\mathrm{Li}$ plating/stripping, the uniform distribution of $\mathrm{LiF}$ in the electrodes was verified by EDS elemental mapping of fluorine (Figure S13). A CE of $97.2 \%$ could be achieved for AP-LiF for 177 cycles at $3 \mathrm{~mA} \mathrm{~cm}^{-2}$ and of $95.7 \%$ for 126 cycles at $10 \mathrm{~mA} \mathrm{~cm}^{-2}$ (Figure S14). Notably, while the CE stability of AP-LiF was lower than that of APCTF-LiF, it was higher than that of the AP due to the SEI stabilization by LiF. Furthermore, as shown in Figure S15, the nucleation overpotentials of AP, AP-LiF and AP-CTF-LiF were 116, 96 and $57 \mathrm{mV}$, respectively. The lower overpotential of AP-LiF compared to that of AP could be ascribed to facile Li diffusion around the LiF boundary. ${ }^{[8 b, 21]}$ More importantly, the lowest overpotential of AP-CTF-LiF reflects the significantly lowered Li plating barrier based on the synergistic effect between CTF and in situ generated LiF. The smallest voltage hysteresis of AP-CTF-LiF (Figure S16) points to the uniform Li distribution owing to the high lithiophilicity of the CTF backbone. To further prove the lithiophilicity of the CTF backbone, we compared the N 1s spectra of CTF before and after $\mathrm{Li}$ plating (Figure $3 \mathrm{~d}$ ). The $\mathrm{N}$ 1s peak shifted from $398.9 \mathrm{eV}$ to $399.5 \mathrm{eV}$ upon Li plating, pointing to the strong N$\mathrm{Li}$ interaction and thus, to the lithiophilicity of the CTF backbone. ${ }^{[22]}$ The structural stability of CTF was verified by FT-IR analysis after 50 cycles, which still showed (Figure S17) the characteristic triazine peak of the AP-CTF-LiF electrode.

AP-CTF-LiF exhibited (Figure $3 \mathrm{e}$ ) an extraordinary cycling stability for 700 cycles when tested at a current density of $5 \mathrm{~mA} \mathrm{~cm}^{-2}$ with an areal capacity of $1 \mathrm{mAh} \mathrm{cm}^{-2}$ 
under Li-Li symmetric cell configuration. The small voltage fluctuation of AP-CTF-LiF along with $36 \mathrm{mV}$ polarization throughout 700 cycles was attributed to a smooth and uniform Li plating/stripping process by the aforementioned synergistic effect. Furthermore, electrochemical impedance spectroscopy (EIS) analysis was conducted to evaluate the interfacial stability and charge transfer kinetics. The plots of fresh cells and after 20 cycles for bare $\mathrm{Cu}, \mathrm{AP}$ and AP-CTF-LiF were fitted to an equivalent circuit (Figure S18 and Table S4). The higher bulk resistances $\left(R_{0}\right)$ of AP and AP-CTF-LiF electrodes before and after cycling in contrast to metallic $\mathrm{Cu}$ were ascribed to the insulating nature of the AP layer. Owing to the uniform Li plating and stable interface formation during cycling, AP-CTF-LiF showed the smallest charge transfer resistance $\left(R_{\mathrm{ct}}\right)$ of $12.82 \Omega$ after 20 cycles. The largest $R_{\mathrm{ct}}$ of AP $(46.32 \Omega)$ reflects a sluggish electrode-electrolyte interface originating from the insulating AP framework. The middle semicircle $(25.49 \Omega)$ for bare $\mathrm{Cu}$ can be explained by thicker SEI layer formation ${ }^{[23]}$ during Li plating/stripping cycles. Lastly, lithium-sulfur (Li-S) cells were evaluated by employing pre-lithiated bare $\mathrm{Cu}, \mathrm{AP}$ and AP-CTF-LiF anodes with an areal capacity of $3 \mathrm{mAh} \mathrm{cm}^{-2}$ (Figure S19a) and pairing with sulfur@graphene cathodes. Owing to the insulating nature of the AP substrate, cells containing AP or AP-CTF-LiF showed lower specific capacities at $1 \mathrm{C}$ compared to those of the bare $\mathrm{Cu}$ counterpart in the early cycling period. The cell based on the AP-CTF-LiF showed higher specific capacity and its retention $(42.1 \%)$ after 375 cycles compared to that of the AP $(28.6 \%)$, in agreement with the $\mathrm{CE}$ and symmetrical cell stability results. In sharp contrast, the cell based on bare $\mathrm{Cu}$ can be cycled (Figure S19b) only for 57 cycles. The cell based on the bare $\mathrm{Cu}$ anode exhibited incomplete charging behavior without reaching the upper cutoff voltage of $2.8 \mathrm{~V}$, unlike AP and AP-CTF-Li. This phenomenon is ascribed to short circuit formation by $\mathrm{Li}$ dendrite growth, which is consistent with the abrupt voltage fluctuations in the cell measurements in Figure 3.

In conclusion, we have introduced a new synthetic strategy for the preparation of CTFs using LiTFSI, which also enabled in situ generation of LiF particles. Importantly, the resulting CTF maintained high nitrogen content, an important parameter for high lithiophilicity. The integration of CTF-LiF with a low-cost, 3D paper led to a synergistic effect of lithiophilicity from the CTF backbone, SEI stabilization from $\mathrm{LiF}$ and sufficient $\mathrm{Li}$ storage space from AP, to suppress dendrite growth and thus, to stabilize Li metal anodes for prolonged cycles. This study demonstrates the possibility of expanding the application of porous organic polymers into emerging battery technologies, particularly where mechanochemical control is needed for long-term charge-discharge reversibility.

\section{Acknowledgements}

A.C. acknowledges financial support by University of Fribourg. J.W.C. acknowledges financial support from a National Research Foundation of Korea grant (NRF2018R1A2A1A19023146 and NRF-2018M1A2A2063340).

\section{Conflict of interest}

The authors declare no conflict of interest.

Keywords: Li metal anodes · LiF · lithiophilicity . porous organic polymers $\cdot$ Li-dendrite growth

How to cite: Angew. Chem. Int. Ed. 2019, 58, 16795-16799 Angew. Chem. 2019, 131, 16951-16955

[1] a) D. Lin, Y. Liu, Y. Cui, Nat. Nanotechnol. 2017, 12, 194-206; b) J. W. Choi, D. Aurbach, Nat. Rev. Mater. 2016, 1, 16013.

[2] a) R. Zhang, N.-W. Li, X.-B. Cheng, Y.-X. Yin, Q. Zhang, Y.-G. Guo, Adv. Sci. 2017, 4, 1600445; b) T.-w. Kwon, J. W. Choi, A. Coskun, Joule 2019, 3, 662-682.

[3] F. Ding, W. Xu, G. L. Graff, J. Zhang, M. L. Sushko, X. Chen, Y. Shao, M. H. Engelhard, Z. Nie, J. Xiao, X. Liu, P. V. Sushko, J. Liu, J.-G. Zhang, J. Am. Chem. Soc. 2013, 135, 4450-4456.

[4] a) D.-J. Yoo, K. J. Kim, J. W. Choi, Adv. Energy Mater. 2018, 8, 1702744; b) A. Basile, A. I. Bhatt, A. P. O'Mullane, Nat. Commun. 2016, 7, 11794.

[5] D.-J. Yoo, S. Yang, Y. S. Yun, J. H. Choi, D. Yoo, K. J. Kim, J. W. Choi, Adv. Energy Mater. 2018, 8, 1802365.

[6] H. Ota, K. Shima, M. Ue, J.-i. Yamaki, Electrochim. Acta 2004, 49, 565-572.

[7] D. Aurbach, E. Pollak, R. Elazari, G. Salitra, C. S. Kelley, J. Affinito, J. Electrochem. Soc. 2009, 156, A694.

[8] a) S. Choudhury, L. A. Archer, Adv. Electron. Mater. 2016, 2, 1500246; b) Y. Yuan, F. Wu, Y. Bai, Y. Li, G. Chen, Z. Wang, C. Wu, Energy Storage Mater. 2019, 16, 411-418.

[9] D. J. Yoo, A. Elabd, S. Choi, Y. Cho, J. Kim, S. J. Lee, S. H. Choi, T.-w. Kwon, K. Char, K. J. Kim, A. Coskun, J. W. Choi, Adv. Mater. 2019, 31, 1901645.

[10] Y. Liu, Q. Liu, L. Xin, Y. Liu, F. Yang, E. A. Stach, J. Xie, Nat. Energy 2017, 2, 17083.

[11] a) L.-L. Lu, J. Ge, J.-N. Yang, S.-M. Chen, H.-B. Yao, F. Zhou, S.H. Yu, Nano Lett. 2016, 16, 4431 - 4437; b) Q. Yun, Y.-B. He, W. Lv, Y. Zhao, B. Li, F. Kang, Q.-H. Yang, Adv. Mater. 2016, 28, 6932 -6939; c) S.-S. Chi, Y. Liu, W.-L. Song, L.-Z. Fan, Q. Zhang, Adv. Funct. Mater. 2017, 27, 1700348.

[12] C. Yang, Y. Yao, S. He, H. Xie, E. Hitz, L. Hu, Adv. Mater. 2017, 29, 1702714.

[13] P. Kuhn, M. Antonietti, A. Thomas, Angew. Chem. Int. Ed. 2008, 47, 3450-3453; Angew. Chem. 2008, 120, 3499-3502.

[14] a) X. Zhu, C. Tian, S. M. Mahurin, S.-H. Chai, C. Wang, S. Brown, G. M. Veith, H. Luo, H. Liu, S. Dai, J. Am. Chem. Soc. 2012, 134, $10478-10484$; b) S. N. Talapaneni, T. H. Hwang, S. H. Je, O. Buyukcakir, J. W. Choi, A. Coskun, Angew. Chem. Int. Ed. 2016, 55, 3106-3111; Angew. Chem. 2016, 128, 3158-3163.

[15] S. Ren, M. J. Bojdys, R. Dawson, A. Laybourn, Y. Z. Khimyak, D. J. Adams, A. I. Cooper, Adv. Mater. 2012, 24, 2357-2361.

[16] K. Wang, L.-M. Yang, X. Wang, L. Guo, G. Cheng, C. Zhang, S. Jin, B. Tan, A. Cooper, Angew. Chem. Int. Ed. 2017, 56, $14149-$ 14153; Angew. Chem. 2017, 129, 14337-14341.

[17] S.-Y. Yu, J. Mahmood, H.-J. Noh, J.-M. Seo, S.-M. Jung, S.-H. Shin, Y.-K. Im, I.-Y. Jeon, J.-B. Baek, Angew. Chem. Int. Ed. 2018, 57, 8438-8442; Angew. Chem. 2018, 130, 8574-8578.

[18] Y. Diao, K. Xie, S. Xiong, X. Hong, J. Electrochem. Soc. 2012, 159, A1816-A1821.

[19] D. E. Arreaga-Salas, A. K. Sra, K. Roodenko, Y. J. Chabal, C. L. Hinkle, J. Phys. Chem. C 2012, 116, $9072-9077$.

[20] K. Schwinghammer, S. Hug, M. Mesch, J. Senker, B. V. Lotsch, Energy Environ. Sci. 2015, 8, 3345-3353.

[21] M. Wang, Z. Peng, W. Luo, F. Ren, Z. Li, Q. Zhang, H. He, C. Ouyang, D. Wang, Adv. Energy Mater. 2019, 9, 1802912. 
[22] a) Y. Guo, P. Niu, Y. Liu, Y. Ouyang, D. Li, T. Zhai, H. Li, Y. Cui, Adv. Mater. 2019, 31, 1900342; b) Z. Lu, Q. Liang, B. Wang, Y. Tao, Y. Zhao, W. Lv, D. Liu, C. Zhang, Z. Weng, J. Liang, H. Li, Q.-H. Yang, Adv. Energy Mater. 2019, 9, 1803186.
[23] Z. Liang, G. Zheng, C. Liu, N. Liu, W. Li, K. Yan, H. Yao, P.-C. Hsu, S. Chu, Y. Cui, Nano Lett. 2015, 15, 2910-2916.

Manuscript received: July 9, 2019

Revised manuscript received: September 1, 2019

Accepted manuscript online: September 17, 2019

Version of record online: October 11, 2019 